\title{
Risikostratifizierung für Therapieentscheidungen bei Rheumatoider Arthritis
}

\section{Risk Stratification for Treatment Decisions in Rheumatoid Arthritis}

\author{
Autoren \\ Katinka Albrecht $\mathbb{D}^{\mathbb{D}}$, Anne Regierer $\mathbb{D}^{\mathbb{D}}$, Anja Strangfeld \\ Institut \\ Deutsches Rheuma-Forschungszentrum, Programmbereich \\ Epidemiologie und Versorgungsforschung, Berlin, \\ Deutschland \\ Schlüsselwörter \\ DMARDs, biologics, disease activity, infections, comorbidity \\ Key words \\ DMARDs, Biologika, Krankheitsaktivität, Infektionen, \\ Komorbidität \\ Online publiziert $\quad 09.02 .2021$ \\ Bibliografie \\ Akt Rheumatol 2021; 46: 143-148 \\ DOI 10.1055/a-1340-0000 \\ ISSN 0341-051X \\ (C) 2021. Thieme. All rights reserved. \\ Georg Thieme Verlag KG, Rüdigerstraße 14, \\ 70469 Stuttgart, Germany \\ Korrespondenzadresse \\ Dr. Katinka Albrecht \\ Deutsches Rheuma-Forschungszentrum, Programmbereich \\ Epidemiologie und Versorgungsforschung \\ Charitéplatz 1 \\ 10117 Berlin \\ Deutschland \\ Tel.: + 49 (0)30 28460-767, Fax: + 49 (0)30 28460-626 \\ albrecht@drfz.de
}

\section{ZUSAMMENFASSUNG}

Um für die Behandlung der rheumatoiden Arthritis unter der Vielzahl an zugelassenen krankheitsmodifizierenden Antirheumatika die geeignete Therapie auszuwählen, werden Patienten anhand ihres Risikos für einen ungünstigen Krankheitsverlauf stratifiziert. Die Auswahl geeigneter Parameter zur Risikostratifizierung werden in dieser Übersichtsarbeit dargestellt. Beim heutigen Therapieziel einer Remission sind hohe Krankheitsaktivität, Funktionseinschränkungen, Begleiterkrankungen und Übergewicht relevante Faktoren für einen ungünstigen Verlauf. Weitere Einflussfaktoren auf die Therapieentscheidung wie Begleiterkrankungen, Infektionsrisiko und spezielle Risikokonstellationen wie vorausgegangene schwerwiegende Infektionen oder Malignome werden in diesem Artikel diskutiert.

\section{ABSTRACT}

In order to select the appropriate drug for the treatment of rheumatoid arthritis, patients are stratified according to their risk of an unfavourable course. This review paper presents the selection of suitable parameters for risk stratification. In the light of the current therapeutic target of remission, relevant factors for an unfavourable course include high disease activity, functional limitations and the presence of comorbidities and obesity. Also this article discusses further factors influencing treatment decisions, e. g. concomitant diseases, risk of infection and special risk constellations such as previous serious infections or malignancies.

\section{Einleitung}

In den letzten Jahren steht eine zunehmende Anzahl gut wirksamer Therapien mit unterschiedlichen Wirkmechanismen für die Behandlung der rheumatoiden Arthritis (RA) zur Verfügung. War früher Methotrexat das Maß aller Dinge, sind heute die Möglichkeiten, im Behandlungsverlauf alternative Subtanzen einzusetzen, deutlich vielfältiger geworden. Biologische disease modifying antirheumatic drugs (bDMARDs) haben die Behandlung der RA revolutioniert, und ein sich schnell entwickelndes Spektrum biologischer Therapien, zu denen jetzt auch Biosimilars gehören, bietet mehr Auswahl und therapeutische Optionen für RA-Patienten als je zuvor.

Neben konventionell synthetischen (cs)DMARDs und Biologika (bDMARDs) sind die Janus-Kinase Inhibitoren Baricitinib, Filgotinib, Tofacitinib und Upadacitinib als targeted synthetic (ts)DMARDs zur Behandlung der RA zugelassen. Mithilfe dieser zahlreichen Therapieoptionen kann die Entzündungsaktivität der RA heute schneller und wirksamer unterdrückt werden. Dadurch hat sich das Therapieziel verschoben. Früher war das Aufhalten der radiologischen Progression der wichtigste Marker, um eine Therapie als wirksam einzustufen. Heute ist das Erreichen einer vollständigen Remission der Arthritis, möglichst bevor radiologische Veränderungen ein- 
treten, oberstes Therapieziel. Kann das nicht erreicht werden, so ist zumindest eine niedrige Krankheitsaktivität anzustreben.

Um für den individuellen Patienten das Medikament auszuwählen, das geeignet ist, das Therapieziel zu erreichen, ist eine NutzenRisiko Stratifizierung notwendig. Hierfür werden unter anderem Charakteristika wie die Höhe der Krankheitsaktivität, das Ausmaß der Funktionseinschränkungen, Begleiterkrankungen und das Alter der Patienten einbezogen, denn nicht jeder Patient benötigt ein $b$ oder tsDMARD und nicht jeder Patient verträgt alle Medikamente gleich gut. Diese Fragen stellen sich im Krankheitsverlauf immer wieder neu.

\section{Was sagen die Leitlinien?}

Relativ komplex geworden sind auch die Therapiealgorithmen der deutschen S2e-Leitlinie zur Therapie der RA mit krankheitsmodifizierenden Medikamenten und der Empfehlungen der European League Against Rheumatism (EULAR) zum Management der RA mit cs oder bDMARDs [1,2]. Bei jeder Visite ist zu überprüfen, ob das Therapieziel Remission oder zumindest eine niedrige Krankheitsaktivität, erreicht ist und, falls nicht, ob der Patient ungünstige Prognosefaktoren aufweist. Ungünstige Prognosefaktoren umfassen laut Leitlinien eine hohe Krankheitsaktivität trotz durchgeführter csDMARD-Therapie, hohe Entzündungsparameter, eine große Anzahl geschwollener Gelenke, der Nachweis von Rheumafaktoren oder anti citrullinated peptide antibodies (ACPA), Nachweis von Erosionen im frühen Erkrankungsverlauf und das Versagen von bereits 2 oder mehr csDMARDs [1, 2]. Anhand dieser Prognosefaktoren wird eine Risikostratifizierung vorgenommen, die bei Vorliegen eine schnellere Therapieintensivierung mit b/tsDMARDs erlaubt. Limitierend für diese Auswahl von Risikofaktoren ist, dass mehrere dieser Marker ursprünglich für das Aufhalten der radiologischen Progression validiert wurden und nicht für das Erreichen einer Remission [3]. Ist Remission das Therapieziel, sollten frühe Erosionen gar nicht erst entstehen. Dies bedeutet, dass die Therapie also bereits zu einem deutlich früheren Zeitpunkt intensiviert werden muss.

\section{Parameter zum Stratifizieren des Risikos}

Aufgrund der Veränderung des Therapieziels wurde anhand von Daten aus dem deutschen Biologika Register RABBIT und der Früharthritiskohorte CAPEA evaluiert, welche Parameter sich auch zum Abschätzen einer ungünstigen Prognose hinsichtlich des Erreichens einer Remission bzw. einer niedrigen Krankheitsaktivität eignen. Von den CAPEA Patienten erreichten nur $39 \%$ unter dem ersten csDMARD und in RABBIT $26 \%$ unter dem zweiten csDMARD bzw. 30\% unter dem ersten TNF-Inhibitor eine Remission nach 6 Monaten, bemessen am DAS28. Eine niedrige Krankheitsaktivität wurde von 45-58\% der Patienten erreicht. Dieser Anteil war deutlich geringer, wenn die Patienten eine hohe Krankheitsaktivität, Funktionseinschränkungen, 2 oder mehr Komorbiditäten aufwiesen oder übergewichtig waren ( $\triangleright$ Tab. 1) [4].

\section{Hohe Krankheitsaktivität}

In den deutschen und internationalen Empfehlungen und Leitlinien ist hohe Krankheitsaktivität übereinstimmend als Parameter zur Risikostratifizierung enthalten [1, 2, 5]. Die Ergebnisse von RABBIT und der CAPEA-Studie bestätigen den hohen Aussagewert der Krankheitsaktivität für das Erreichen einer Remission. 53 \% der Früharthritispatienten aus CAPEA hatten vor Beginn der ersten csDMARD Therapie eine hohe Krankheitsaktivität, bemessen am DAS28 [4]. In den Empfehlungen des American College of Rheumatology (ACR) von 2015 wird für die Therapieentscheidung sogar ausschließlich nach Krankheitsaktivität stratifiziert [5]. Zur Bestimmung der Krankheitsaktivität können neben dem DAS28 auch andere Instrumente wie der Simplified Disease Activity Index (SDAI) oder der Clinical Disease Activity Index (CDAI) verwendet werden. Bei letzterem ist ein aktueller Laborwert bei der Visite nicht unbedingt notwendig.

\section{Funktionseinschränkungen}

Eine Einschränkung der Funktionskapazität der Gelenke hat für den Patienten im Gegensatz zu frühen Erosionen direkte Auswirkungen auf die berufliche und soziale Teilhabe. Neben CAPEA und RABBIT zeigten sich auch in der französischen Früharthritiskohorte ESPOIR Funktionseinschränkungen als unabhängiger Prädiktor für das Nichterreichen von Remission [6]. Funktionseinschränkungen eignen sich gut für die Risikostratifizierung, da sie mithilfe von Patientenfragebögen wie dem Funktionsfragebogen Hannover (FFbH) oder dem Health Assessment Questionnaire (HAQ) ohne großen Aufwand identifiziert werden können [7].

\section{Komorbidität}

Begleiterkrankungen werden zunehmend als Risikomarker für den Krankheitsverlauf berücksichtigt. In den RABBIT-Daten waren es vor allem Patienten mit 2 oder mehr Begleiterkrankungen, die seltener eine Remission oder niedrige Krankheitsaktivität erreichten [4]. Begleiterkrankungen sind darüber hinaus mit weiteren Faktoren assoziiert, die wiederum den Krankheitsverlauf ungünstig beeinflussen. Muskuloskeletale Komorbiditäten wie Arthrose oder Osteoporose können ihrerseits die physische Funktion beeinflussen, mit der Folge eingeschränkter Beweglichkeit und verminderter sportlicher Betätigung. Komorbiditäten gehen meist auch mit zusätzlicher Medikamenteneinnahme einher, die mit den antirheu-

- Tab. 1 Risikofaktoren für Nicht-Erreichen von Remission oder niedriger Krankheitsaktivität nach [4].

Hohe Krankheitsaktivität (DAS28 > 5,1)

Funktionseinschränkungen: $\mathrm{HAQ} \geq 1,2$ oder $\mathrm{FFbH}<70$

$\geq 2$ Komorbiditäten

Übergewicht $\left(\mathrm{BMI}>30 \mathrm{~kg} / \mathrm{m}^{2}\right)$

DAS28 Disease Activity Score mit 28 Gelenken, HAQ Health Assessment Questionnaire, FFbH Funktionsfragebogen Hannover, BMI Body Mass Index 
matisch wirksamen Substanzen interagieren. Und nicht zuletzt erhöhen Komorbiditäten, insbesondere wenn sie nicht adäquat behandelt sind, das Risiko von unerwünschten Ereignissen unter einer Therapie der RA [8, 9].

In den letzten Jahren ist die Depression als relevante Komorbidität in den Fokus gerückt. Bezüglich der Therapiewahl ist sie von Bedeutung, weil eine begleitende Depression bei RA das Therapieziel, die Therapieadhärenz, den Krankheitsverlauf und die Mortalität beeinflussen kann. Die Wahrscheinlichkeit, eine Remission zu erreichen, ist bei Vorliegen einer Depression geringer, da diese mit einer höheren Krankheitslast, stärkeren Schmerzen und einer höheren Zahl schmerzhafter Gelenke assoziiert ist [10]. Bei der Therapiewahl muss bei Patienten mit RA und einer Depression also einerseits eine ausreichend wirksame Therapie gewählt werden, um die pro-inflammatorische Aktivität als Trigger der Depression zu senken, andererseits muss das vermeintlich fehlende Therapieansprechen hinsichtlich der patientenrelevanten Messinstrumente berücksichtigt werden. Der klinische Befund und die Entzündungsmarker sind hier zur Evaluierung geeignet, da sie von der Depression weniger beeinflusst sind.

\section{Infektionsrisiko}

Bei vergleichbarer Wirksamkeit verschiedener bDMARDs ist das Infektionsrisiko ein ausschlaggebender Parameter für die Auswahl des am besten geeigneten bDMARD. Verschiedene Studien haben gezeigt, dass unter Tumor Nekrose Faktor (TNF) Inhibitoren und anderen Biologika häufiger schwerwiegende Infektionen auftreten als unter csDMARD Therapie $[11,12]$. Auf der anderen Seite ist eine Exposition gegenüber Biologika bei Beginn einer schwerwiegenden Infektion signifikant mit einem selteneren Auftreten einer Sepsis und verminderter Mortalität assoziiert [13]. Relevante Parameter, die das Infektionsrisiko erhöhen, sind höheres Alter, Komorbiditäten, wiederholte Therapieversagen, schwerwiegende Infektionen in der Anamnese und Medikation mit Glukokortikoiden [11]. Dosis, Häufigkeit und Dauer der Glukokortikoid-Therapie sind ausschlaggebende Faktoren bei der Risikostratifizierung bezüglich Infektionen bei RA. Der RABBIT Risiko Score ist eine Möglichkeit, das individuelle Risiko für den Patienten zu ermitteln [14]. Der Score berechnet die Wahrscheinlichkeit eines Patienten mit RA, innerhalb der nächsten 12 Monate eine schwerwiegende Infektion zu erleiden. Er ist online frei verfügbar (https://biologika-register.de/

- Tab. 2 Risikofaktoren für schwerwiegende Infektion unter DMARD Therapie nach [14].

Alter $>60$ Jahre

Funktionseinschränkungen: $\mathrm{FFbH}<70$

Schwerwiegende Infektion in den letzten 12 Monaten

COPD oder andere chronische Lungenerkrankung

Chronische Nierenerkrankung

Anzahl abgesetzter DMARDs $\geq 5$

Glukokortikoide $\geq 7,5 \mathrm{mg}$ Tagesdosis

DMARD disease modifying antirheumatic drug, FFbH Funktionsfragebogen Hannover, COPD chronic obstructive pulmonary disease rabbit/risikoscore-fuer-infektionen/) und umfasst die Parameter, die das Infektionsrisiko relevant beeinflussen ( $\triangleright$ Tab. 2).

Atemwegs, Haut- und Weichteil-, Urogenital- und Knochen-/ Gelenkinfektionen sind die häufigsten Infektionen, die unter bDMARD Therapien auftreten. Für die meisten schwerwiegenden Infektionen haben Studien keine relevanten Unterschiede in der Häufigkeit bei den verschiedenen bDMARDs gezeigt, nachdem für Patientencharakteristika adjustiert wurde [11]. Daten aus dem britischen Biologikaregister zeigten ein erhöhtes Risiko von schwerwiegenden Infektion bei mit Tocilizumab behandelten Patienten im Vergleich zu Etanercept [15]. Letztlich bleiben vergleichende Auswertungen des Infektionsrisikos unter verschiedenen bDMARDs aber schwierig, weil nicht $100 \%$ ig für Patientencharakteristika und die unterschiedlichen Voraussetzungen, die zu einer Therapiewahl geführt haben, adjustiert werden kann. Es gibt aber ein paar Situationen, die dafür sprechen, bestimmte Wirkstoffgruppen bevorzugt einzusetzen.

Bei Patienten mit Risikofaktoren für eine latente Tuberkulose (Tbc) ist das Risiko einer Reaktivierung unter Etanercept geringer als unter Infliximab oder Adalimumab, sodass in diesem Fall Etanercept die bevorzugte Wahl unter den TNF Inhibitoren ist [11]. Risikofaktoren für eine latente Tbc sind anamnestische Tbc, Kontakt zu aktiver Tbc, Geburt oder > dreimonatiger Aufenthalt in Tbc prävalenten Regionen oder Aufenthalt in Einrichtungen mit erhöhter Tbc Prävalenz.

Das Risiko einer Herpes Zoster Infektion erscheint unter Januskinase Inhibitoren deutlich erhöht, v. a. wenn zusätzlich Glukokortikoide eingenommen werden [16]. Dies sollte bei der Therapiewahl, vor allem bei Patienten mit erhöhtem Risiko für eine Herpes zoster Infektion (Herpes zoster Anamnese, hohes Alter, Glukokortikoide> 7,5mg/Tag) berücksichtigt werden.

Möglichkeiten, das Infektionsrisiko zu minimieren und trotzdem ein b/tsDMARD einsetzen zu können, sind Screening (z. B. auf Tuberkulose und Hepatitis), begleitende antivirale/antibakterielle Therapie, Impfungen (u. a. gegen Pneumokokken, Influenza, Herpes Zoster) und Patientenaufklärung bezüglich Risikoverhaltens ( Tab. 3).

\section{Therapieentscheidung bei Risikokonstellationen}

\section{Vorausgegangene schwerwiegende Infektionen}

Patienten mit vorausgegangenen schwerwiegenden Infektionen haben ein erhöhtes Risiko für eine erneute Infektion. Es gibt aber Evidenz, dass bei Patienten, die unter einem TNF Inhibitor eine

Tab. 3 Risikominimierung von Infektionen unter DMARD Therapie.

Screening auf Hepatitis, Tuberkulose

Ggf. antivirale/antibakterielle Therapie

Impfung gegen Influenza, Pneumokokken, Herpes Zoster

Screening und Therapie kardiovaskulärer Komorbidität

Aufklärung bezüglich Risikoverhalten 
schwerwiegende Infektion hatten, das Risiko für eine erneute Infektion größer war, wenn die Therapie beendet wurde, als wenn der TNF Inhibitor fortgesetzt oder auf einen anderen Wirkstoff umgestellt wurde [17]. Gleichermaßen ist belegt, dass eine hohe Krankheitsaktivität das Risiko einer Infektion deutlich erhöht [18].

\section{Kardiovaskuläre Komorbidität}

Gleiches gilt für Patienten mit kardiovaskulären Erkrankungen. Sie profitieren zweifach von einer effektiven DMARD-Therapie, da die konsequente Senkung der Krankheitsaktivität auch das Risiko für kardiovaskuläre Ereignisse deutlich reduziert. Für Methotrexat und für TNF Inhibitoren ist eine Reduktion des kardiovaskulären Risikos in mehreren Studien nachgewiesen und erste Studien legen eine ähnlich protektive Wirksamkeit für nicht-TNF-Biologika nahe [19]. Wichtig ist, dass neben einer adäquaten DMARD Therapie auch die kardiovaskuläre Begleiterkrankung medikamentös eingestellt ist. Zwei Studien aus RABBIT haben gezeigt, dass sowohl eine hohe Krankheitsaktivität der RA, als auch eine fehlende medikamentöse Therapie der kardiovaskulären Komorbidität das Risiko für einen Schlaganfall oder Herzinfarkt deutlich erhöhen [8,9]. Bei Patienten mit RA und Herzinsuffizienz ab NYHA-Grad 3 galten alle TNFInhibitoren außer Etanercept lange als kontraindiziert. Die ACR Empfehlungen von 2015 sprechen sich bei Vorliegen einer Herzinsuffizienz für eine Bevorzugung von csDMARDS, anderen bDMARDS oder Tofacitinib gegenüber TNF Inhibitoren aus [5]. Daten aus RABBIT haben gezeigt, dass unter TNF-Inhibitoren in Normaldosierung kein erhöhtes Risiko bei Vorliegen einer Herzinsuffizienz besteht, sondern möglicherweise durch effektive Senkung der Krankheitsaktivität sogar das Risiko reduziert wird [20]. Diese Annahme wurde kürzlich durch eine Metaanalyse bestätigt, wenngleich es weiterhin einen Mangel an qualitativ hochwertigen Studien zu diesem Thema gibt [21].

\section{Malignomanamnese}

Therapieentscheidungen bei Patienten mit Malignomanamnese sind eine Herausforderung. Da eine unkontrolliert hohe Krankheitsaktivität der RA ein wichtiger Risikofaktor für weitere Komorbiditäten und eine verkürzte Lebensdauer ist, wird bei anamnestischen Malignomen eine effektive DMARD Therapie benötigt, ohne dass ein höheres Risiko für ein Wiederauftreten des Malignoms in Kauf genommen werden muss. Langzeitregisterdaten deuten inzwischen mehrheitlich darauf hin, dass unter der Behandlung mit TNF Inhibitoren und Rituximab kein erhöhtes Risiko für das Auftreten einer Krebserkrankung besteht [22]. Zwei kollaborative Auswertungen europäischer Biologika-Register haben keinen Anhalt für ein erhöhtes Risiko für Lymphome und Melanome unter bDMARDTherapie gezeigt [23, 24]. Bezüglich des Rezidivrisikos vorheriger Malignome unter TNF Inhibitoren und Rituximab gibt es aus Registerdaten keine Hinweise auf eine Erhöhung [25, 26], so dass die Patienten entsprechend ihrem klinischen Bedarf behandelt werden sollten [27]. In der Praxis wird bei Patienten mit vorausgegangenem Malignom Rituximab häufiger eingesetzt, während der Anteil an Patienten mit anderen bDMARDs mit 1-6\% nach einer aktuellen Auswertung der skandinavischen Register sehr gering ist [28]. Der zurückhaltende Einsatz von bDMARDs kann eine unzureichende Senkung der Krankheitsaktivität bei vielen Patienten bedeuten und unterstreicht die Notwendigkeit, weitere Daten zur Sicherheit bei Malignomanamnese, insbesondere für die anderen b und tsDMARDs, zu generieren.

\section{Therapieentscheidung nach Lebenssituation}

Ob ein Patient jung oder alt, berufstätig oder in Rente ist, eine Familie versorgen muss, sich selbst versorgen kann oder pflegebedürftig ist, entscheidet neben den vielen anderen Faktoren ebenfalls über die geeignete Therapie. Darüber hinaus verschiebt sich in unterschiedlichen Lebenssituationen unter Umständen das Therapieziel. Bei jungen Patienten mit früher RA ist das Erreichen einer Remission bei vollem Funktionserhalt oberstes Therapieziel. Eine kurzfristige Therapie mit Glukokortikoiden ist bei diesen Patienten überdies mit einem geringeren Risiko behaftet als bei Patienten mit begleitendem Diabetes oder einer Osteoporose. Bei älteren Patienten mit irreversiblen Funktionsseinschränkungen ist eine uneingeschränkte Funktionskapazität unerreichbar, der Erhalt der bestehenden Restfunktion aber elementar.

Auch Lifestyle-Faktoren können für die Therapiestratifizierung herangezogen werden. Eine aktuelle Studie aus RABBIT hat gezeigt, dass bei übergewichtigen Patienten die Senkung der Krankheitsaktivität unter Therapien, deren Wirkungsweise auf der Zytokinhemmung beruht, deutlich schwächer ausfiel als bei Therapien, die auf die Zellebene oder Zell-Interaktionen abzielen [29]. Eine geringere Verbesserung im DAS28 zeigte sich v. a. bei übergewichtigen Frauen unter TNF-Inhibitoren und Tocilizumab. Unter Rituximab und Abatacept hatte Übergewicht keinen Einfluss auf die Wirksamkeit.

\section{Risiko einer unzureichenden rheumatologischen Versorgung}

Letztlich muss auch das Risiko für eine fehlende oder unregelmäßige rheumatologische Versorgung in der Therapieplanung berücksichtigt werden. Juvenile Patienten, die im Übergang ins Erwachsenenalter eine neue rheumatologische Betreuung suchen müssen, schwangere Patientinnen und multimorbide bzw. zunehmend pflegebedürftige Patienten sind Beispiele, die ein höheres Risiko für eine Unterbrechung oder einen kompletten Abbruch der rheumatologischen Versorgung haben [30-32]. Im Idealfall kann dies bei der Visite besprochen oder durch eine auf die Situation angepasste Therapie vermieden werden. Bei dem einen Patienten kann das eine regelmäßige Infusion und damit Vorstellung in der Praxis sein, bei dem anderen eine eigenständig durchzuführende subkutane Applikation und bei dem dritten können es Tabletten sein, die auch der Hausarzt verschreiben kann.

\section{Zusammenfassung}

Um eine Risikostratifzierung für die Therapieentscheidungen vorzunehmen, muss zunächst das Therapieziel festgelegt werden. Im Idealfall ist es das Erreichen einer Remission, bei Vorliegen von Vorerkrankungen oder anderen besonderen Umständen und auch im Alter kann dies auch anders definiert werden. Die Wahl der Therapie richtet sich in den heutigen Leitlinien auch nach dem Vorliegen von ungünstigen Prognosefaktoren. Es gibt aber individuelle Besonderheiten, die ein Abweichen von den Empfehlungen durchaus rechtfertigen. Für die Therapieentscheidung ist die individuelle Nutzen-Risiko Abschätzung für jeden Patienten unter Berücksichtigung der genannten Parameter notwendig. 


\section{Interessenkonflikt}

Die Autorinnen/Autoren geben an, dass kein Interessenkonflikt besteht.

\section{Literatur}

[1] Fiehn C, Holle J, Iking-Konert C et al. S2e-Leitlinie: Therapie der rheumatoiden Arthritis mit krankheitsmodifizierenden Medikamenten. Z Rheumatol 2018; 77: 35-53. doi:10.1007/s00393-018-0481-y

[2] Smolen JS, Landewe RBM, Bijlsma JW] et al. EULAR recommendations for the management of rheumatoid arthritis with synthetic and biological disease-modifying antirheumatic drugs: 2019 update. Ann Rheum Dis 2020; 79: 685-699. doi:10.1136/annrheumdis-2019-216655

[3] Albrecht K, Zink A. Poor prognostic factors guiding treatment decisions in rheumatoid arthritis patients: a review of data from randomized clinical trials and cohort studies. Arthritis Res Ther 2017; 19: 68. doi:10.1186/s13075-017-1266-4

[4] Baganz L, Richter A, Albrecht K et al. Are prognostic factors adequately selected to guide treatment decisions in patients with rheumatoid arthritis? A collaborative analysis from three observational cohorts. Semin Arthritis Rheum 2019; 48: 976-982. doi:10.1016/j.semarthrit.2018.09.003

[5] Singh JA, Saag KG, Bridges SL Jr. et al. 2015 American College of Rheumatology Guideline for the Treatment of Rheumatoid Arthritis. Arthritis Rheumatol 2016; 68: 1-26. doi:10.1002/art.39480

[6] Castrejon I, Dougados M, Combe B et al. Prediction of Remission in a French Early Arthritis Cohort by RAPID3 and other Core Data Set Measures, but Not by the Absence of Rheumatoid Factor, Anticitrullinated Protein Antibodies, or Radiographic Erosions. J Rheumatol 2016; 43: 1285-1291. doi:10.3899/jrheum. 141586

[7] Lautenschlager ], Mau W, Kohlmann T et al. Vergleichende Evaluation einer deutschen Version des Health Assessment Questionnaires (HAQ) und des Funktionsfragebogens Hannover (FFbH). Z Rheumatol 1997; 56: 144-155. doi:10.1007/s003930050030

[8] Meissner Y, Zink A, Kekow J et al. Impact of disease activity and treatment of comorbidities on the risk of myocardial infarction in rheumatoid arthritis. Arthritis Res Ther 2016; 18: 183. doi:10.1186/ s13075-016-1077-z

[9] Meissner $Y$, Richter A, Manger B et al. Serious adverse events and the risk of stroke in patients with rheumatoid arthritis: results from the German RABBIT cohort. Ann Rheum Dis 2017; 76: 1583-1590. doi:10.1136/annrheumdis-2017-211209

[10] Michelsen B, Kristianslund EK, Sexton J et al. Do depression and anxiety reduce the likelihood of remission in rheumatoid arthritis and psoriatic arthritis? Data from the prospective multicentre NOR-DMARD study. Ann Rheum Dis 2017; 76: 1906-1910. doi:10.1136/annrheumdis-2017-211284

[11] Jani M, Barton A, Hyrich K. Prediction of infection risk in rheumatoid arthritis patients treated with biologics: are we any closer to risk stratification? Curr Opin Rheumatol 2019; 31: 285-292. doi:10.1097| BOR.0000000000000598

[12] Strangfeld A, Eveslage M, Schneider M et al. Treatment benefit or survival of the fittest: what drives the time-dependent decrease in serious infection rates under TNF inhibition and what does this imply for the individual patient? Ann Rheum Dis 2011; 70: 1914-1920. doi:10.1136/ard.2011.151043

[13] Richter A, Listing J, Schneider M et al. Impact of treatment with biologic DMARDs on the risk of sepsis or mortality after serious infection in patients with rheumatoid arthritis. Ann Rheum Dis 2016; 75: 1667-1673. doi:10.1136/annrheumdis-2015-207838
[14] Zink A, Manger B, Kaufmann J et al. Evaluation of the RABBIT Risk Score for serious infections. Ann Rheum Dis 2014; 73: 1673-1676. doi:10.1136/annrheumdis-2013-203341

[15] Rutherford Al, Subesinghe S, Hyrich KL et al. Serious infection across biologic-treated patients with rheumatoid arthritis: results from the British Society for Rheumatology Biologics Register for Rheumatoid Arthritis. Ann Rheum Dis 2018; 77: 905-910. doi:10.1136/annrheumdis-2017-212825

[16] Curtis JR, Xie F, Yang S et al. Risk for Herpes Zoster in Tofacitinib-Treated Rheumatoid Arthritis Patients With and Without Concomitant Methotrexate and Glucocorticoids. Arthritis Care Res (Hoboken) 2019; 71: 1249-1254. doi:10.1002/acr.23769

[17] Subesinghe S, Rutherford Al, Byng-Maddick R et al. Biologic prescribing decisions following serious infection: results from the British Society for Rheumatology Biologics Register-Rheumatoid Arthritis. Rheumatology (Oxford) 2018; 57: 2096-2100. doi:10.1093/ rheumatology/key198

[18] Au K, Reed G, Curtis JR et al. High disease activity is associated with an increased risk of infection in patients with rheumatoid arthritis. Ann Rheum Dis 2011; 70: 785-791. doi:10.1136/ard.2010.128637

[19] Krüger K, Nusslein H. Kardiovaskuläre Komorbiditäten bei rheumatoider Arthritis. Z Rheumatol 2019; 78: 221-227. doi:10.1007/ s00393-018-0584-5

[20] Listing J, Strangfeld A, Kekow J et al. Does tumor necrosis factor alpha inhibition promote or prevent heart failure in patients with rheumatoid arthritis? Arthritis Rheum 2008; 58: 667-677. doi:10.1002/ art.23281

[21] Baniaamam M, Paulus W], Blanken AB et al. The effect of biological DMARDs on the risk of congestive heart failure in rheumatoid arthritis: a systematic review. Expert Opin Biol Ther 2018; 18: 585-594 doi:10.1 080/14712598.2018.1462794

[22] Wadstrom H, Frisell T, Askling J et al. Malignant Neoplasms in Patients With Rheumatoid Arthritis Treated With Tumor Necrosis Factor Inhibitors, Tocilizumab, Abatacept, or Rituximab in Clinical Practice: A Nationwide Cohort Study From Sweden. JAMA Intern Med 2017; 177: 1605-1612. doi:10.1001/jamainternmed.2017.4332

[23] Mercer LK, Askling J, Raaschou P et al. Risk of invasive melanoma in patients with rheumatoid arthritis treated with biologics: results from a collaborative project of 11 European biologic registers. Ann Rheum Dis 2017; 76: 386-391. doi:10.1136/annrheumdis-2016-209285

[24] Mercer LK, Regierer AC, Mariette X et al. Spectrum of lymphomas across different drug treatment groups in rheumatoid arthritis: a European registries collaborative project. Ann Rheum Dis 2017; 76: 2025-2030. doi:10.1136/annrheumdis-2017-211623

[25] Silva-Fernandez L, Lunt M, Kearsley-Fleet $L$ et al. The incidence of cancer in patients with rheumatoid arthritis and a prior malignancy who receive TNF inhibitors or rituximab: results from the British Society for Rheumatology Biologics Register-Rheumatoid Arthritis. Rheumatology (Oxford) 2016; 55: 2033-2039. doi:10.1093/ rheumatology/kew314

[26] Raaschou P, Frisell T, Askling J et al. TNF inhibitor therapy and risk of breast cancer recurrence in patients with rheumatoid arthritis: a nationwide cohort study. Ann Rheum Dis 2015; 74: 2137-2143. doi:10.1136/annrheumdis-2014-205745

[27] Regierer AC, Strangfeld A. Rheumatoid arthritis treatment in patients with a history of cancer. Curr Opin Rheumatol 2018; 30: 288-294. doi:10.1097/BOR.0000000000000492

[28] Chatzidionysiou K, Delcoigne B, Frisell T et al. How do we use biologics in rheumatoid arthritis patients with a history of malignancy? An assessment of treatment patterns using Scandinavian registers. RMD Open 2020; 6 (2): e001363. doi:10.1136/rmdopen-2020-001363 
[29] Schäfer M, Meissner Y, Kekow J et al. Obesity reduces the real-world effectiveness of cytokine-targeted but not cell-targeted disease-modifying agents in rheumatoid arthritis. Rheumatology (Oxford) 2020; 59: 1916-1926. doi:10.1093/rheumatology/kez535

[30] Luque Ramos A, Hoffmann F, Albrecht K et al. Transition to adult rheumatology care is necessary to maintain DMARD therapy in young people with juvenile idiopathic arthritis. Semin Arthritis Rheum 2017; 47: 269-275. doi:10.1016/j.semarthrit.2017.05.003
[31] Luque Ramos A, Redeker I, Hoffmann F et al. Comorbidities in Patients with Rheumatoid Arthritis and Their Association with Patient-reported Outcomes: Results of Claims Data Linked to Questionnaire Survey. J Rheumatol 2019; 46: 564-571. doi:10.3899/jrheum.180668

[32] Luque Ramos A, Albrecht K, Zink A et al. Rheumatologic care of nursing home residents with rheumatoid arthritis: a comparison of the year before and after nursing home admission. Rheumatol Int 2017; 37: 2059-2064. doi:10.1007/s00296-017-3791-5 